\title{
The Analysis of the related performance during MICRO-EDM
}

\author{
Liu shuyang, Han Zhihong \\ Jingdezhen Ceramic Institute, Jingdezhen 333403, China \\ Corresponding author: Dr. Liu shuyang ,Tel:+86-187-97883058; \\ e-mai: Hanliuyang@163.com
}

\begin{abstract}
In this paper, the energy on workpiece surface in electrical discharge machining (short in EDM) process were modeled and its related parameters were analysized, based on electron sputtering and the resistance heat reaction of current flow when the tool electrode is negative. The study shows that: when the steady-state discharge current is constant, workpiece surface of power energy concentration is higher.The proportion of resistance heat reaction on workpiece surface in

total power energy $\eta_{c}^{+}$decreases with the increase of the equivalent radius ratio of real discharging area to the area of workpiece end surface $\lambda$, and when $\lambda$ is constant , $\eta_{c}^{+}$ increases with the increase of $J_{t 3}$. The experimental phenomena and results verify the rationality and effectiveness of the proposed model.
\end{abstract}

Keywords-Resistance heat reaction;Electron spilling work; Electron sputtering work

\section{INTRODUCTION}

As a kind of special machining technology, EDM has already been indispensable in processing ultra hard materials and heat sensitive materials[1-3]. However electrical discharge phenomena in EDM make both observation and theoretical analysis extremely difficult. Therefore, some questions are still remained. First, the material removal mechanisms are not yet well understood. Second, what are the relationships between the energy distribution and working efficiency? Third, how the EDM process results in the energy distribution [4]? It shows that further research work is necessary for a better understanding of the nature of the phenomena involved in the EDM process.

Many scholars' research and related experiments have already shown that particles in the discharge channel were mainly on electric streams ejected from cathode, and the effects of positive ions on the channel were very small[5]. Modern field-emission theories and thermal field emission theories, especially F-N equation descriptions, demonstrated that electrons escaping from surface needed to overcome surface barriers to work, which would translate into heat energy ,and gave the reactive functions of material[6-8]. $\mathrm{Mao}, \mathrm{C}$ et al. conducted that with longer pulse-on time, the power available between the anode and cathode becomes greater, thus strengthening discharge energy [9]。 LIU et al. [10] derived electrodes complied with skin effect when flowing through the electrodes, which gave space boundary conditions for energy analysis of material removal.

In EDM discharge cycle, a variety of signs indicate that a large number polarity ion accumulation exists in interelectrode channel. Many scholars' research has shown that the most of the material removal was mainly caused by free electrons emitted from cathode. And the melting trace on the discharge surface in EDM show that heat effect was the main work mechanism of material removal, but the surface topography difference on anode and cathode surface further explained that work energy sources and methods were different. Modern field-emission theory about cold and heat electron emission introduced and perfected

In this paper, the energies on workpiece surface both in positive polarity polarity EDM process were modeled and its related parameters were analyzed, based on electron sputtering and the resistance heat reaction of current flow when the tool electrode is negative. A set of machining blind-hole experiments with pole electrodes were carried out and investigated, and the related parameters that affect the working efficiencies were discussed. The experimental phenomena and results verify the rationality and effectiveness of the proposed model.

\section{THE SINGLE-HOLE EDM EXPERIMENT WITH POLE ELECTRODES}

In order to analyses the mechanism and parameters of EDM processing, we designed single-hole EDM experiments with pole electrodes shown in Fig .1, where $S w_{p}$ represents the pulse switch and $U_{s}$ is the voltage of discharging tube, $R_{p p}$ and $R_{n p}$ are the resistances of tool electrode and workpiece electrode respectively, $R_{p v}$ is the resistance of single discharging tube, and the amount of pulse tubes is $n$. 


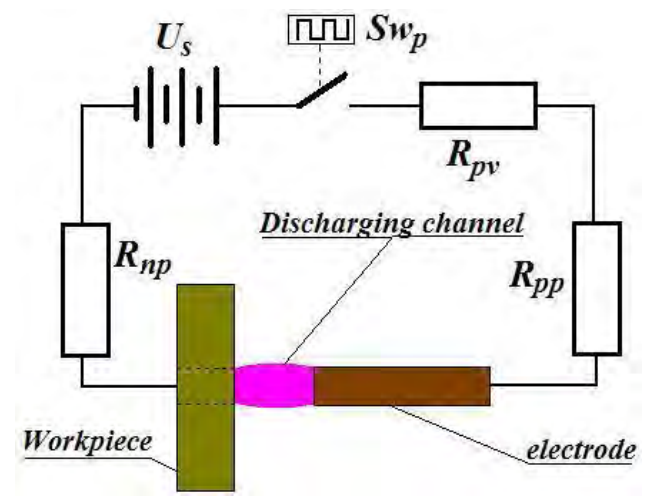

Figure 1. Single-hole EDM experiment with pole electrode
Related parameters of devices applied in the experiments are as follows:

Machine model: $A G I E \quad$ CHARMILLES SE2

Tool electrode: $45^{\#}$ steel, $D=8 m m, L=40 m m$

Workpiece electrode: $45^{\#}$ steel, $D=80 \mathrm{~mm}, L=3.5 \mathrm{~mm}$

Inputting power: $P_{i n}=10 \mathrm{kw}$;

Dielectric medium: kerosene

relevant technical details and results of experiments are shown in table 1.

TABLE. 1 TECHNICAL DETAILS OF THE SINGLE-HOLE EDM EXPERIMENTS

\begin{tabular}{|c|c|c|c|c|c|c|c|c|c|c|c|c|c|}
\hline \multirow[t]{2}{*}{ Z } & \multirow{2}{*}{$\begin{array}{l}\overline{0} \\
\stackrel{0}{2} \\
\stackrel{2}{*}\end{array}$} & \multicolumn{2}{|c|}{ Material } & \multicolumn{3}{|c|}{$\begin{array}{l}\text { Discharge } \\
\text { parameters }\end{array}$} & \multicolumn{2}{|c|}{$\begin{array}{l}\text { Lift-put } \\
\text { cycle }\end{array}$} & \multirow[b]{2}{*}{$\mathrm{n}$} & \multirow{2}{*}{ 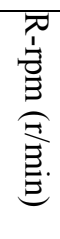 } & \multirow{2}{*}{ 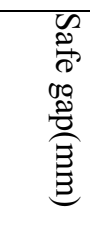 } & \multirow[b]{2}{*}{ EDM time } & \multirow[b]{2}{*}{ mean time } \\
\hline & & 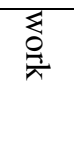 & $\begin{array}{l}\overrightarrow{0} \\
\stackrel{0}{0}\end{array}$ & $\stackrel{\overrightarrow{\mathscr{E}}}{\stackrel{\vec{E}}{E}}$ & $\begin{array}{c}\overrightarrow{0} \\
\stackrel{0}{\tilde{E}_{0}}\end{array}$ & 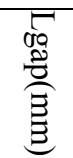 & $\frac{\overrightarrow{7}}{\sqrt{\frac{\pi}{6}}}$ & $\frac{\vec{\lambda}}{\hat{C}}$ & & & & & \\
\hline 1 & \multirow{3}{*}{-} & \multirow{3}{*}{$45 \#$} & \multirow{3}{*}{$45 \#$} & 10 & 5.6 & 0.08 & 0.8 & 0.6 & 7 & 0 & 0.18 & $472 \mathrm{~h} 53^{\prime}$ & \multirow{3}{*}{$523 \mathrm{~h} 31^{\prime}$} \\
\hline 2 & & & & 10 & 5.6 & 0.08 & 0.8 & 0.6 & 7 & 0 & 0.18 & $562 \mathrm{~h} 05^{\prime}$ & \\
\hline 3 & & & & 10 & 5.6 & 0.08 & 0.8 & 0.6 & 7 & 0 & 0.18 & 535h34' & \\
\hline
\end{tabular}

III. HE WORK ENERGY ANALYSIS ON THE WORKPIECE SURFACE OF NEGATIVE EDM PROCESS

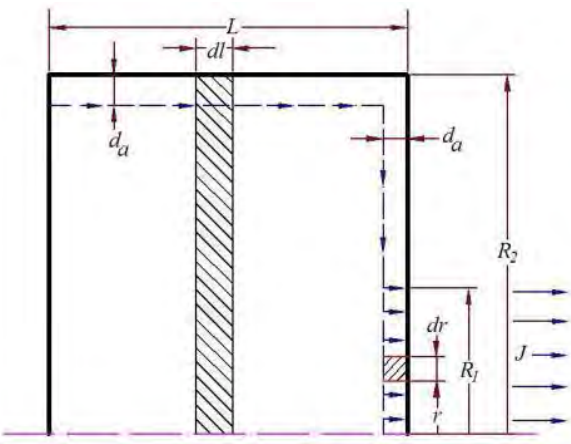

Figure 2. the movement of electron current in workpiece electrode

The foregoing experiments indicated that during the negative EDM process, the work energy on the workpiece surface also has two origins, one is the sputtering energy

$Q_{h}$ produced by the high-speed field-emission electrons, and the other is the uniform resistance heat $Q_{s}$ generated by electrons current flowing through the limited workpiece surface.

According to the Skin Effect of electric charge, the electron current channel performs a shape as a concentric tube with two diameters $2 \pi\left(R_{2}-d_{a}\right) \times L$ and $\pi\left(R_{2}{ }^{2}-r^{2}\right) \times d_{a}$ respectively, shown in Fig .2. Where, $A_{1}$ is equal to the end area of interelectrode discharging channel, $A_{2}$ is the superficial area of workpiece, $J_{t}$ is the density of the electron current at the $t$ moment, $d_{a}$ is nearly equal to the mean diameter of atoms of workpiece material, $R_{1}$ is the radius of area $A_{1}$ which is equal to the equivalent radius of the discharging channel, and $R_{2}$ is the equivalent radius of area because the discharging channel between electrode pairs are vacuum during the steady discharging stage, so it was considered that there is no energy loss for the field-emission electrons when they are high-speed moving in the interelectrode channel, and all kinetic energy carried by them is completely acted on the machining surface. Supposing the initial speed of fieldemission electrons away from the surface of tool electrode is zero and according to the law of conservation of energy, the $Q_{h}$ should be equal to the potential energy of fieldemission electrons at the moment when they just break through the surface barriers of tool electrode, id est. the sputtering energy during a micro period $d t$ of steady discharging stage can be expressed as. 


$$
d Q_{h}^{A_{1}^{+}}=d E_{e}=U \cdot J_{t 3} \cdot A_{1} \cdot d t
$$

By integral, thus the sputtering energy during the steady discharging stage of a discharging pulse can be obtained,

$$
Q_{h}^{A_{1}^{+}}=U \cdot \pi R_{1}^{2} \cdot J_{t 3} \cdot\left(T_{o n}-t_{3}\right)
$$

Same to the expression of the Eq. (12), the total

resistance heat on $A_{1}^{+}$area during a single discharging pulse can be calculated as,

$$
Q_{s}{ }^{\left(A_{1}^{+}\right)}=Q_{s}{ }^{\left(A_{1}^{-}\right)}=\frac{2 \rho_{w}}{3 \pi \cdot d_{a}} \cdot\left(\frac{R_{2}}{R_{1}}-\frac{R_{1}^{2}}{R_{2}{ }^{2}}+\frac{3}{4}\right) \cdot\left(T_{\text {on }}-t_{3}\right)
$$

So the total work energy on the area of ${ }^{A_{1}^{+}}$during a single discharge pulse can be obtained, at some certain $\lambda, \eta_{c}^{+}$gets higher with the increase of $J_{t 3}$.

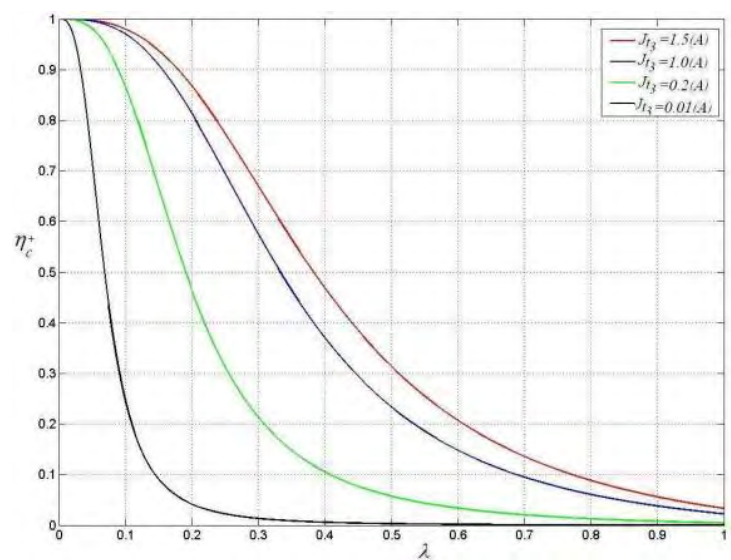
$Q^{\left(A_{1}^{+}\right)}=Q_{h}^{A_{1}^{+}}+Q_{s}^{\left(A_{1}^{+}\right)}=U \cdot \pi R_{1}^{2} \cdot J_{t 3} \cdot\left(T_{o n}-t_{3}\right)+\frac{2 \rho_{w}}{3 \pi \cdot d_{a}} \cdot\left(\frac{R_{2}}{R_{1}}-\frac{R_{1}^{2}}{R_{2}{ }^{2}}+\frac{3}{4}\right) \cdot J_{t 3}{ }^{2} \cdot\left(T_{o n}-t_{3}\right)$ the varying curves of $\eta_{C}^{+}$about $\lambda$ at different $J_{t 3}$

Ditto defining the ratio of the resistance heat to the total work energy on the area of $A_{1}$ as,

$$
\eta_{c}^{+}=\frac{Q_{s}^{\left(A_{1}^{+}\right)}}{Q^{A_{1}^{+}}}
$$

Substituting the Esq. (3) and (4) in the equation above, then the ratio of the resistance heat to the total work energy during the steady discharging stage can be gotten as,

$$
\eta_{c}^{+}=\frac{\frac{2 \rho_{w}}{3 \pi \cdot d_{a}} \cdot\left(\frac{R_{2}}{R_{1}}-\frac{R_{1}^{2}}{R_{2}{ }^{2}}+\frac{3}{4}\right) \cdot J_{t 3}}{U \cdot \pi R_{1}^{2}+\frac{2 \rho_{w}}{3 \pi \cdot d_{a}} \cdot\left(\frac{R_{2}}{R_{1}}-\frac{R_{1}^{2}}{R_{2}^{2}}+\frac{3}{4}\right) \cdot J_{t 3}}
$$

Defining $C_{3}=U \cdot \pi$, then the equation above can be simplified by the substitution of $C_{1}, C_{2} C_{3}$ and $\lambda$ as,

$$
\eta_{c}^{+}=\frac{C_{2} \cdot\left(4-4 \lambda^{3}+3 \lambda\right) \cdot J_{t 3}}{C_{3} \cdot \lambda^{3} \cdot R_{2}^{2}+C_{2} \cdot\left(4-4 \lambda^{3}+3 \lambda\right) \cdot J_{t 3}}
$$

In our study, the pulse voltage of AGIE CHARMILLES SE2 machine is $U=110(\mathrm{~V})$ so, $C_{3}=345.575(V)$

Taking the data of the single-hole EDM experiment of negative polarity listed in table. 1 as the example too, the varying tendency of $\eta_{C}^{+}$about $\lambda$ at different steady discharging current are shown in Fig .3. Obviously, $\eta_{c}^{+}$is an increasing function about $\lambda$ at certain $J_{t 3}$, and

\section{CONCLUSIONS}

(1) Based on electron sputtering and the resistance heat reaction of current flow, the work energy on the machined surface in negative polarity EDM process was studied, and the relevant equations were given。The related parameters that affect the working efficiencies were discussed.

(2) The analysis of the combined machining method as well as experimental research has resulted in an empirical relation which highlights the influence of some of the analyzed work conditions 。 It can be obtained that the ratio of the resistance heat to the total work energy $\eta_{c}^{+}$increase with the decrease of the equivalent radius ratio of real discharging area and the area of workpiece end surface $\lambda$ when $J_{t 3}$ is a constant and $\eta_{c}^{+}$would increase with the increase of the interelectrode discharging current when $\lambda$ is a constant

\section{REFERENCES}

[1] V. Yadav, V.K. Jain, P.M. Dixit ,Thermal stresses due to electrical discharge machining, International Journal of Machine Tools \& Manufacture ,2002,42 : 877-888.

[2] R.Pérez, J.Carron, M. Rappaz, G. Wälder, B. Revaz, R. Flükiger. Measurement and metallurgical modeling of the thermal impact of EDM discharges on steel, in: Proceedings of the 15th International Symposium on Electromachining, ISEM $\mathrm{XV}, 2007:$ 17-22. 
[3] M.S. Murali, S. H. Yeo. Process simulation and residual stress estimations of micro-electrodischarge machining using finite element method, Japanese Journal of Applied Physics,2005, 44 (7A) : 5254-5263.

[4] B. Lauwers, J.P. Kruth, W. Liu, W. Eeraerts, B. Schacht, P. Bleys, Investigation of material removal mechanism in EDM of composite ceramic materials. Mater. Process. Technol. 149 (2004) 347-352.

[5] Shuyang Liu,Yumei Huang,Yan Li.A Plate capacitor model of the EDM process based on the field emission theory. International, Journal of Machine Tools and Manufacture2011,51(7):653-659.

[6] R. H. Fowler, L. W. Nordheim. Electron emission in intense electric fields, [J]. roc. Roy. Soc. Lund. Ser. A $119,(1928) 173$.
[7] E L. Murphy, R. H. Good. Thermionic emission . field emission. and the transition region, Phys. Rev. 102, (1956)1464.

[8] R. H. Hare, R. M. Hill, and C. J. Budd. Modeling charge injection and motion in solid dielectrics under high electric field. Plays. D : Appl. Phys. 26, (1993)1084.

[9] Lin, Y.C.; Chen, Y.F.; Lin, C.T.; Tzeng, H.J. Electrical discharge machining (EDM) characteristics associated with electrical discharge energy on machining of cemented tungsten carbide. Materials and Manufacturing Processes 2008, 23 (3-4), 391-399.

[10] LIU Yu, ZHAO Fulin etc. Influences of skin-effect on microEDM. Journal of Dalian University of Technology, 2010(3):362-367. 